\title{
Infección por Cyclospora cayetanensis. Revisión a propósito de tres casos de diarrea del viajero
}

\author{
Juan C. Weitz V., Carolina Weitz R., Marilena Canales R. y Ruy Moya R.
}

\section{Cyclospora cayetanensis infection. Updated review a propos of three cases of traveler's diarrhea}

Human infection by Cyclospora cayetanensis, namely cyclosporiasis, can cause a wide range of symptoms in immunocompetent patients, from mild to severe diarrhea. Immunocompromised patients can present with chronic diarrhea and it has been recognized as a cause of traveler's diarrhea. We report three patients who traveled from Chile to Peru, who presented upon returning with prolonged traveler's diarrhea. A literature review about cyclosporiasis is presented, with emphasis on the clinical, epidemiological, diagnostic and therapeutic aspects of this disease.

Key words: Traveler's diarrhea, diarrhea, cyclosporiasis, Cyclospora, Cyclospora cayetanensis

Palabras clave: Diarrea del viajero, diarrea, ciclosporosis, Cyclospora, Cyclospora cayetanensis.

\section{Introducción}

C yclospora cayetanensis es un protozoo coccidio, que fue descrito inicialmente como una cianobacteria o un tipo de coccidio (coccidian like bodyCLB) productor de diarrea aguda. Los primeros casos se publicaron entre 1977 y $1978^{1}$, hasta que finalmente fue caracterizado por Ortega y cols en $1993^{2}$.

Desde entonces se ha reconocido a este agente como productor de diarrea aguda y prolongada en inmunocompetentes, de diarrea del viajero, como causa de numerosos brotes epidemiológicos y de diarrea crónica en pacientes inmunocomprometidos.

El objetivo de esta comunicación es describir tres casos de diarrea del viajero por C. cayetanensis y revisar la literatura científica publicada hasta la fecha.

\section{Casos clínicos}

Caso 1. Hombre de 28 años, chileno, con antecedente de haber viajado a la ciudad de Oroya (Sierra central del Perú). Siete días después de su regreso a Chile presentó diarrea, sin mucus, pus ni sangre, con una frecuencia de cuatro veces al día, durante dos semanas. Consultó en la tercera semana, cuando la frecuencia de las deposiciones había aumentado a más de 10 veces durante el día y dos en la noche. Relató como síntomas acompañantes: dolor abdominal difuso intenso, calofríos y mialgias. Al examen físico estaba afebril y levemente deshidratado.
En el examen abdominal se encontró ruidos hidro-aéreos aumentados en frecuencia y leve sensibilidad difusa a la palpación. Se diagnosticó diarrea del viajero prolongada, y se solicitó: coprocultivo, leucocitos fecales y examen parasitológico seriado de deposiciones con tinción de Ziehl Neelsen. Se le indicó que, una vez tomadas las muestras de deposiciones, iniciara tratamiento con ciprofloxacina $500 \mathrm{mg}$ cada 12 horas por cinco días. Los exámenes fueron negativos excepto la tinción de Z. Neelsen en deposiciones, que informó ooquistes de C. cayetanensis. El paciente completó el tratamiento indicado y relató disminución de la diarrea y el dolor abdominal al segundo día. Al recibir el resultado positivo para C. cayetanensis (tres días luego de finalizar el tratamiento con ciprofloxacina) se le indicó cotrimoxazol 160/800 mg cada 12 h durante siete días, con resolución completa del cuadro. El paciente no se efectuó examen de control solicitado.

Caso 2. Hombre de 38 años, chileno, con antecedentes de trabajar una semana al mes en Lima, Perú. Consultó por padecer, al regreso de Lima, diarrea intensa afebril, de siete días de evolución, con una frecuencia de más de cuatro veces al día, acompañada de dolor abdominal leve difuso. El examen físico era normal. Se le indicó dos cápsulas de Saccharomyces boulardii diarias por tres días, y se solicitó coprocultivo, leucocitos fecales y parasitológico seriado con Z. Neelsen. A los 10 días, el paciente mantenía diarrea de similar frecuencia, con mayor dolor abdominal y meteorismo. La tinción de Z. Neelsen mostró
Integramédica, Santiago, Chile

Departamento de Gastroenterología (JCWV, RMR)

Laboratorio (MCR)

Universidad de Los Andes,

Santiago, Chile

Facultad de Medicina

Estudiante de pre-grado (CWR)

Universidad Católica de Chile, Santiago

Facultad de Medicina

Laboratorios clínicos Laboratorio

Parasitología (MCR)

Recibido: 16 de marzo de 2009

Aceptado: 6 de septiembre de 2009

Correspondencia a: Juan C. Weitz Vattuone jcweitz@gmail.com 
ooquistes de $C$. cayetanensis en deposiciones. Se le trató con cotrimoxazol (160/800) cada 12 h durante 7 días, con resolución de sus síntomas.

Caso 3. Mujer de 50 años, chilena, que trabajaba en forma estable en Lima, Perú y viajaba a Chile en forma esporádica. Consultó por padecer 20 días de síntomas caracterizados por náuseas intensas, vómitos ocasionales, ardor epigástrico y diarrea cinco veces al día, sin mucus, pus ni sangre. El examen físico era normal. Se le indicaron medidas generales, se solicitó coprocultivo y leucocitos fecales negativos, y el Z. Neelsen en deposiciones mostró ooquistes de $C$. cayetanensis. Se trató con cotrimoxazol $(160 / 800)$ cada 12 horas por 10 días. Los controles no mostraron presencia de protozoos en la tinción de $\mathrm{Z}$. Neelsen.

Las muestras de los tres pacientes fueron procesadas en el laboratorio de Integramédica y leídas por uno de los autores (MCR). Los diagnósticos fueron corroborados por el Laboratorio de Referencia de Parasitología del Instituto de Salud Pública de Chile.

Ninguno de los pacientes presentaba síntomas ni antecedentes sugerentes de inmunocompromiso y no se estudió esta condición por la buena respuesta al tratamiento.

\section{Discusión}

El agente. Cyclospora cayetanensis es un protozoo coccidio que pertenece al phyllum Apicomplexa, y a la suborden Eimeriida, al igual que Toxoplasma gondii, Cryptosporidium sp e Isospora belli. Su nombre se debe al Dr. Schneider, quien lo descubrió en intestinos de animales y lo denominó Cyclospora en 1870, y cayetanensis por la Universidad Cayetano Heredia (Lima, Perú) donde el Dr. Ortega realizó sus investigaciones ${ }^{2}$.

Existen varias especies de Cyclospora (cercopitheci, colobi, papionis, schneideri, etc), y aparentemente la única que afecta a los humanos es $C$. cayetanensis, no habiéndose comprobado que es una zoonosis. Este protozoo, intracelular obligado, posee un ciclo biológico muy similar al de Cryptosporidium sp, se ingiere el ooquiste (forma infectante), luego tienen lugar reproducciones asexuada y sexuada en el epitelio del intestino delgado, que dan origen a un ooquiste inmaduro, a diferencia de Cryptosporidium, que mide entre 8 y $10 \mu \mathrm{m}$ de diámetro y es expulsado en las deposiciones. Este ooquiste debe permanecer varios días en ambiente favorable (temperatura y humedad) para madurar; por lo tanto, no es capaz de infectar persona a persona. El mecanismo de infección es la ingesta de ooquistes maduros presentes en agua, vegetales o alimentos contaminados y el periodo de incubación promedio es 7 días $^{3-4}$. El ciclo se puede revisar con mayor detalle en la página del CDC de Atlanta 4 .
Epidemiología. Existe una gran variedad de publicaciones, pocos comparables entre sí y que estudian desde muestras de laboratorio hasta pacientes con infección por VIH/SIDA y diarrea crónica. Resumiremos, por lo tanto, las más relevantes.

Estudios de niños sin diarrea muestran frecuencias de $11,9 \%$ en comunidades indígenas de Venezuela ${ }^{5} ; 2,5$ a $22,5 \%$ en niños haitianos (prevalencia ligada a estacionalidad $)^{6}$ y $9 \%$ en niños desnutridos en Guatemala ${ }^{7}$.

La prevalencia de C. cayetanensis en niños con diarrea aguda es de 6 a $18 \%$ en Perú ${ }^{2} ; 5,3 \%$ en niños venezolanos de zona rural ${ }^{8} ; 4,4 \%$ en $\mathrm{Cuba}^{9} ; 4,8 \%$ en $\mathrm{Nepal}^{10}$ y de $2,5 \%$ en niños de Tanzania ${ }^{11}$.

Los estudios de muestras de deposiciones de pacientes con síntomas digestivos presentan una frecuencia de 0,3 a $0,7 \%$ en Sao Paulo, Brasil ${ }^{12-13} ; 0,28 \%$ en un laboratorio de Massachusetts (E.U.A.) ${ }^{14}$ y $0,05 \%$ en tres laboratorios de Ontario, Canadá ${ }^{15}$.

Los estudios de seguimiento de poblaciones son escasos, existe una cohorte en niños peruanos de origen rural que mostró que $33 \%$ de ellos sufrieron diarrea por C. cayetanensis en 37 meses de seguimiento ${ }^{16}$. En Nepal se estudió por un año a 77 extranjeros residentes, quienes sufrieron 3,3 diarrea/persona-año y del total, 11\% fue por este agente ${ }^{17}$. Y en Java (Indonesia), 558 extranjeros fueron seguidos por tres años, y 11,8\% de las diarreas las causó $C$. cayetanensis ${ }^{18}$.

Las prevalencias en pacientes con infección por VIH/ SIDA y diarrea crónica, de trabajos no comparables, varían de 1,1 a $22 \% \%^{7,19-20}$. En nuestra experiencia, no detectamos ooquistes de C. cayetanensis al estudiar 89 casos de infección por VIH/SIDA y diarrea crónica ${ }^{21}$.

La estacionalidad de la infección es un hecho constante en las poblaciones que se han seguido en el tiempo, siendo muy variables de país en país, e incluso dentro de distintas regiones de un mismo país, dependiendo de la humedad y las temperaturas correspondientes.

Clínica. La ciclosporosis, así como otras infecciones intestinales, puede manifestarse con molestias mínimas hasta un cuadro de gran gravedad. En los inmunocompetentes generalmente se presenta con fiebre menor de $38^{\circ} \mathrm{C}$ por uno a dos días, náuseas y vómitos ocasionales, y una diarrea de variable intensidad, con promedio de cinco a siete deposiciones en el día. La duración del cuadro es de cinco a 14 días, pudiendo en algunos casos prolongarse por un mes o más. En los niños puede manifestarse como diarrea aguda y, en algunos casos, prolongada ${ }^{3,22}$.

Si bien se describen muchos casos de diarrea prolongada en los brotes epidémicos y en casos de diarrea del viajero, la ciclosporosis es poco frecuente como etiología de diarrea crónica en inmunocompetentes ${ }^{23}$.

En el paciente inmunocomprometido, especialmente con infección por VIH/SIDA, al igual que las infecciones 
por Cryptosporidium sp. e I. belli, C. cayetanensis produce una diarrea crónica de intensidad variable ${ }^{19}$. Además, puede afectar otros tejidos como la vía biliar, produciendo colecistitis alitiásica y se ha descrito como uno de los agentes involucrados en la colangiopatía del SIDA ${ }^{24}$. En varios casos existen infecciones concomitantes con otros coccidios o con microsporidios

\section{Diarrea del viajero}

Motivados por los tres casos antes descritos, que corresponden a diarreas del viajero, al igual que el primer caso descrito en Chile ${ }^{25}$, revisamos la literatura médica contenida en las bases de datos de PubMed, Lilacs y Google academic (1977-2009).

Los estudios de frecuencia de C. cayetanensis en diarreas del viajero son escasos, y las prevalencias varían de $1,1 \%$ en turistas alemanes ${ }^{26}$ a $4 \%$ en holandeses que retornaban del trópico ${ }^{27}$.

Respecto de casos clínicos, logramos revisar 19 comunicaciones de sólo un paciente y cinco artículos con dos casos reportados. En la Tabla 1 se resumen las publicaciones con tres o más casos con esta patología, incluyendo los definidos como cianobacteria (antes de su caracterización). Estas comunicaciones son muy disímiles en cuanto a las características clínicas de los pacientes y por ello resumimos lo más relevante en-38. $^{28}$.

En resumen, la ciclosporosis es una causa muy infrecuente de diarrea del viajero, se han comunicado $\sim 190$ casos, que en su mayoría corresponden a personas que han viajado a países en desarrollo, y pocos artículos consignan la condición de infección por VIH/SIDA de los turistas. El cuadro se ha manifestado al regresar a su país, con diarrea de más de siete días y varias de ellas cumplen con el criterio de prolongada. Se acompañan de dolor abdominal de intensidad variable, fiebre, náuseas y vómitos, y algunos casos han referido malestar epigástrico y pirosis. Sintomatología similar a la descrita por nuestros casos clínicos.

\section{Brotes epidémicos}

Existen múltiples brotes epidémicos de infección por C. cayetanensis descritos en distintos países del mun$\mathrm{do}^{1,39-42}$, los de mayor importancia se han comunicado en Norteamérica (E.U.A. y Canadá), que sólo en la década de los noventa suman más de 3.500 pacientes en 177 brotes $^{1,39}$.

La mayoría de ellos se ha debido al consumo de vegetales crudos como frambuesas y otros berries, albahaca y ensaladas crudas (mezclas de vegetales). Estos trabajos tienen un gran valor, pues ha contribuido a conocer mejor el período de incubación, los aspectos clínicos y las fuentes de infección de la ciclosporosis.
Tabla 1. Diarrea del viajero por Cyclospora cayetanensis, resumen de trabajos

\begin{tabular}{|c|c|c|c|c|c|}
\hline n casos & País visitado & VIH/SIDA & Síntomas & Duración & Referencia \\
\hline 7 & $\begin{array}{l}\text { Sudamérica } \\
\text { Caribe } \\
\text { Méjico } \\
\text { India } \\
\text { Asia }\end{array}$ & 4 & Diarrea & 14 días & 25 \\
\hline 3 & Pakistán & NC & $\begin{array}{l}\text { Diarrea } \\
\text { Baja peso } \\
\text { Náuseas } \\
\text { Anorexia }\end{array}$ & 14 días & 26 \\
\hline 11 & $\begin{array}{l}\text { Asia } \\
\text { Santo Domingo }\end{array}$ & NC & $\begin{array}{l}\text { Diarrea } \\
\text { Esteatorrea } \\
\text { Náuseas } \\
\text { Fiebre }\end{array}$ & $\begin{array}{l}7 \mathrm{~d} \text { a } 6 \\
\text { sem. }\end{array}$ & 27 \\
\hline 5 & $\begin{array}{l}\text { Méjico } \\
\text { Tailandia }\end{array}$ & 1 & $\begin{array}{l}\text { Diarrea } \\
\text { Dolor abdominal } \\
\text { Vómitos } \\
\text { Fiebre }\end{array}$ & $\begin{array}{l}10 \text { a } 45 \\
\text { días }\end{array}$ & 28 \\
\hline 3 & $\begin{array}{l}\text { Nepal } \\
\text { Pakistán }\end{array}$ & NC & Diarrea & NC & 29 \\
\hline 5 & $\begin{array}{l}\text { Méjico } \\
\text { Guatemala } \\
\text { Tailandia } \\
\text { India }\end{array}$ & NC & Diarrea & $\begin{array}{l}11 \text { a } 32 \\
\text { días }\end{array}$ & 23 \\
\hline 20 & $\begin{array}{l}\text { Centroamérica } \\
\text { India } \\
\text { Indonesia } \\
\text { Malasia } \\
\text { Turquía } \\
\text { Malasia } \\
\text { Gabón }\end{array}$ & NC & $\begin{array}{l}\text { Diarrea } \\
\text { Astenia } \\
\text { Dolor abdominal } \\
\text { Baja de peso } \\
\text { Fiebre } \\
\text { Náuseas/vómitos }\end{array}$ & $\begin{array}{l}10 \text { a } 120 \\
\text { días }\end{array}$ & 30 \\
\hline 32 & Indonesia & NC & $\begin{array}{l}\text { Diarrea } \\
\text { Dolor abdominal } \\
\text { Astenia } \\
\text { Anorexia } \\
\text { Fiebre } \\
\text { Baja de peso }\end{array}$ & $\begin{array}{l}10 \text { a } 30 \\
\text { días }\end{array}$ & 31 \\
\hline 55 & $\begin{array}{l}\text { África } \\
\text { Centro-sudamérica } \\
\text { Turquía } \\
\text { Sub continente indio } \\
\text { Sudeste asiático }\end{array}$ & $\mathrm{NC}$ & $\begin{array}{l}\text { Diarrea } \\
\text { Dolor abdominal } \\
\text { Flatulencia } \\
\text { Baja de peso } \\
\text { Fiebre } \\
\text { Náuseas/vómitos }\end{array}$ & $\begin{array}{l}15 \text { a > } \\
30 \text { días }\end{array}$ & 32 \\
\hline 7 & Guatemala & NC & $\begin{array}{l}\text { Diarrea } \\
\text { Astenia } \\
\text { Flatulencia } \\
\text { Dolor abdominal } \\
\text { Pirosis } \\
\text { Náuseas/vómitos } \\
\text { Baja de peso }\end{array}$ & NC & 33 \\
\hline 6 & $\begin{array}{l}\text { Asia } \\
\text { Madagascar }\end{array}$ & $\mathrm{NC}$ & $\begin{array}{l}\text { Diarrea } \\
\text { Baja de peso }\end{array}$ & NC & 34 \\
\hline 7 & Cuba & No & $\begin{array}{l}\text { Diarrea } \\
\text { Astenia } \\
\text { Flatulencia } \\
\text { Dolor abdominal } \\
\text { Fiebre } \\
\text { Pirosis } \\
\text { Náuseas } \\
\text { Baja de peso }\end{array}$ & $\begin{array}{l}11 \text { a } 28 \\
\text { días }\end{array}$ & 35 \\
\hline 3 & Perú & No & $\begin{array}{l}\text { Diarrea } \\
\text { Dolor abdominal } \\
\text { Calofríos } \\
\text { Ardor epigástrico }\end{array}$ & $\begin{array}{l}10 \text { a } 30 \\
\text { días }\end{array}$ & Weitz y col. \\
\hline
\end{tabular}

VIH/SIDA (virus de inmunodeficiencia humana, síndrome de inmunodeficiencia adquirida) NC: no consignado 
Figura 1. Ooquistes de $C$. cayetanensis con tinción de Z Neelsen, aumento 1000x.

\section{Diagnóstico}

Existen múltiples técnicas de diagnóstico que incluyen desde algunas simples a otras de gran complejidad. Desde el punto de vista clínico, lo más adecuado es solicitar tinciones alcohol-resistente como Ziehl Neelsen modificado (caliente) o Kinyoun (similar, pero en frío). Estas técnicas se aplican a frotis de deposición, recolectadas y concentradas en el procesamiento del parasitológico de deposiciones (tres muestras). Estas tinciones son útiles para la pesquisa de C. cayetanensis, además de I. belli y Cryptosporidium $\mathrm{sp}^{43-44}$. La Figura 1 corresponde a un frotis teñido con $Z$. Neelsen de uno de nuestros pacientes, en que se observa un ooquiste de C. cayetanensis, esférico, acidófilo, de 8 a $10 \mu \mathrm{m}$ de diámetro. El diagnóstico diferencial es con el ooquiste de Cryptosporium, muy similar de forma, acidófilo, pero de 4 a $6 \mu \mathrm{m}$ de diámetro; y con I. belli, cuyo ooquiste es fusiforme, mide 20 a 23 $\mu \mathrm{m}$ de largo, la pared externa delimita la precipitación del colorante y él o los esporoquistes redondeados se tiñen de rojo intenso ${ }^{3}$. La sensibilidad es similar al teñir los frotis con safranina ${ }^{45}$. El ooquiste de Cyclospora, a diferencia de los otros coccidios mencionados, posee
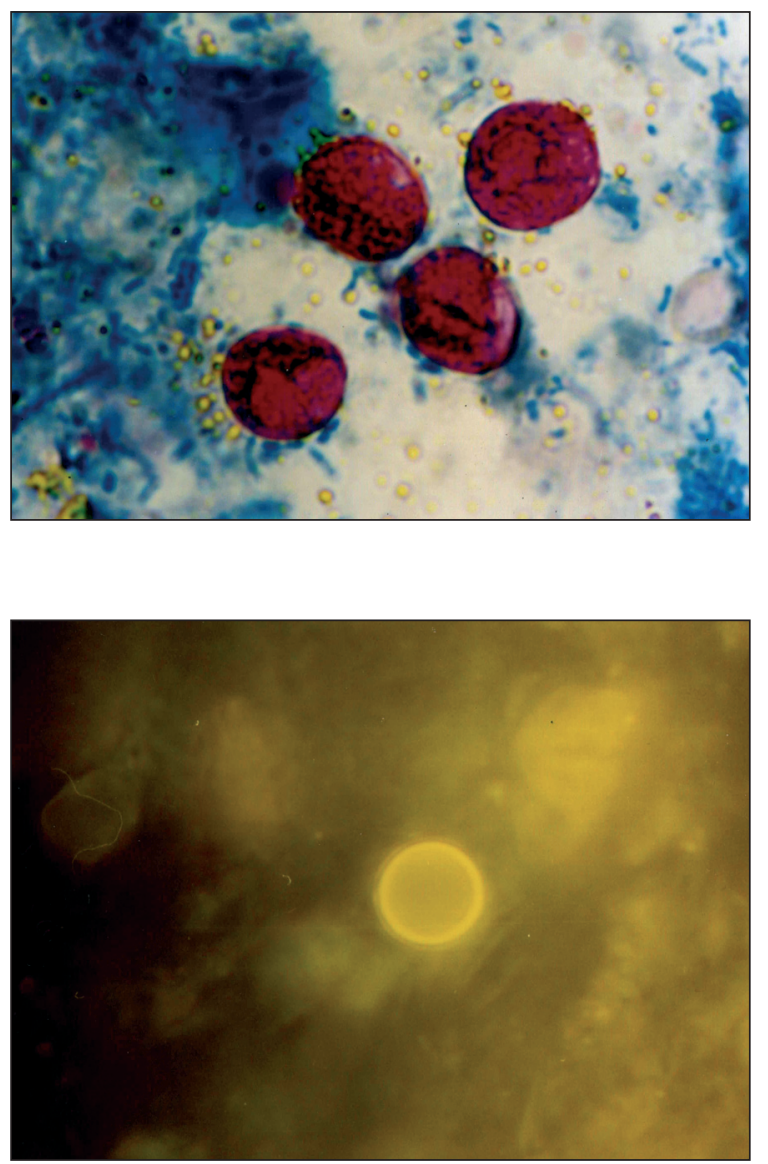

autofluorescencia al observar muestras húmedas con microscopio fluorescente (Figura 2).

\section{Tratamiento}

El tratamiento indicado para el adulto es cotrimoxazol ( $160 \mathrm{mg} / 800 \mathrm{mg}) 2$ veces al día; y en los niños trimetoprim $8 \mathrm{mg} / \mathrm{kg}$-sulfametoxazol $40 \mathrm{mg} / \mathrm{kg}$ fraccionado en dos dosis al día, en ambos grupos por 7 a 10 días. En pacientes con infección por VIH/SIDA se sugiere tratar hasta la desaparición de los síntomas o el agente en deposiciones ${ }^{46}$.

Como alternativa se ha usado ciprofloxacina $500 \mathrm{mg}$ dos veces al día durante una semana, con resultados algo menores que con sulfas (87 versus $100 \%$ ), demostrado en un trabajo comparativo (cotrimoxazol versus ciprofloxacina), efectuado en pacientes con infección por VIH/ SIDA $^{47}$.

Dos de nuestros pacientes fueron tratados con cotrimoxazol una vez conocido el diagnóstico, y al primer paciente le fue indicado empíricamente ciprofloxacina con mejoría importante del cuadro y posteriormente con cotrimoxazol, al recibir el resultado de los exámenes. La respuesta al tratamiento fue satisfactoria en todos los pacientes.

En pacientes con alergia a sulfas o con fracaso al tratamiento con fármacos antes descritos, se ha comunicado buen resultado con nitazoxanida, antiparasitario de amplio espectro (protozoos y algunos helmintos), no disponible aún en nuestro país ${ }^{48}$.

\section{Recomendación}

En pacientes con diarrea del viajero prolongada solicitar además de coprocultivo y leucocitos fecales, tinción de Ziehl Neelsen para buscar Cyclospora cayetanensis.

Recomendaciones generales a todo viajero que visite países con saneamiento ambiental deficiente.

\section{Resumen}

La infección humana por Cyclospora cayetanensis, denominada ciclosporosis, es capaz de producir una amplia gama de síntomas en el inmunocompetente, desde síntomas mínimos a una diarrea aguda que puede llegar a ser grave; en el inmunocomprometido produce diarrea crónica, y se ha reconocido como una de las causas de diarrea del viajero. En esta comunicación se describen tres casos de chilenos que viajaron al Perú y que a su regreso presentaron una diarrea del viajero prolongada. Se efectúa una revisión bibliográfica de la ciclosporosis, enfatizando los aspectos clínicos, epidemiológicos, de diagnóstico y tratamiento.
Figura 2. Ooquiste de $C$ cayetanensis con fluorescencia directa. 


\section{Referencias}

1.- Herwaldt B. Cyclospora cayetanensis: A review, focusing on the outbreaks of cyclosporiasis in the 1990s. Clin Infect Dis 2000; 31: 1040-57.

2.- Ortega Y, Sterling C, Gilman R, Cama V, Díaz F. Cyclospora species-a new protozoan pathogen of humans. N Engl J Med 1993; 328 : 1308-12.

3.- Atías A. Ciclosporosis. En Atías A., Parasitología Médica, Ed. Mediterráneo, Santiago, Chile 1998, págs. 152-5.

4.- Cyclosporiasis. Centers for Disease Control and Prevention, Atlanta. Parasites and health. Disponible en www.dpd.cdc.gov/dpdx/HTML/ Cyclosporiasis.htm (accedido 15-3-09)

5.- Devera R, Blanco Y, Cabello E. Elevada prevalencia de Cyclospora cayetanensis en indígenas del estado Bolívar, Venezuela. Cad Saúde Pública 2005; 21: 1778-84.

6.- López A, Bendik J, Alliance J, Roberts J, da Silva A, Moura I, et al. Epidemiology of Cyclospora cayetanensis and other intestinal parasites in a community in Haiti. J Clin Microbiol 2003; 41: 2047-54.

7.- Pratdesaba R, González M, Piedrasanta E, Mérida C, Contreras K, Vela C, et al. Cyclospora cayetanensis in three populations at risk in Guatemala. J Clin Microbiol 2001; 39: 2951-3.

8.- Chacín-Bonilla L, Estévez J, Monsalve F, Quijada L. Cyclospora cayetanensis infections among diarrheal patients from Venezuela. Am J Trop Med Hyg 2001; 65: 351-4.

9.- Núñez F, González O, González I, Escobedo A, Cordoví R. Intestinal coccidia in Cuban pediatric patients with diarrhea. Mem Inst Oswaldo Cruz 2003; 98: 539-42.

10.- Hoge C, Echeverría P, Rajah R, Jacobs J, Malthouse S, Chapman E, et al. Prevalence of Cyclospora species and other enteric pathogens among children less than 5 years of age in Nepal. J Clin Microbiol 1995; 33: 3058-60.

11.- Cegielski J, Ortega Y, McKee S, Madden J, Gaido L, Schwartz D, et al. Cryptosporidium, Enterocytozoon, and Cyclospora infections in pediatric and adult patients with diarrhea in Tanzania. Clin Infect Dis 1999; 28: 314-21.

12.- Messias do Nascimento E, Horroiva I, Pagliusi V, Pereira C. Estudo retrospectivo da ocorrência de Cyclospora cayetanensis no Hospital das Clínicas da Faculdade de Medicina da Universidade de São Paulo, SP. Rev Soc Bras Med Trop 2005; 38: 326-30.

13.- Alarcón R, Amato Neto V, Gakiya E, Bezerra R. Observações sobre Blastocystis hominis e Cyclospora cayetanensis em exames parasitológicos efetuados rotineiramente. Rev Soc Bras Med Trop 2007; 40: 253-5.

14.- Ooi W, Zimmerman S, Needham C. Cyclospora species as a gastrointestinal pathogen in immunocompetent hosts. J Clin Microbiol 1995; 33: 1267-9.

15.- Brennan B, MacPherson D, Palmer J, Keystone J. Cyclosporiasis: a new cause of diarrhea. Can Med Assoc J 1996; 155: 1293-6.

16.- Bern C, Ortega Y, Checkley W, Roberts J, Lescano A, Cabrera L, et al. Epidemiologic differences between cyclosporiasis and cryptosporidiosis in Peruvian children. Emerg Infect Dis 2002; 8: 581-5.

17.- Shlim D, Hoge C, Rajah R, Scott R, Pandy P, Echeverría P. Persistent high risk of diarrhea among foreigners in Nepal during the first 2 years of residence. Clin Infect Dis 1999; 29: 613-6.

18.- Fryauff D, Krippner R, Prodjodipuro P, Ewald C, Kawengian S, Pegelow K, et al. Cyclospora cayetanensis among expatriate and indigenous populations of West Java, Indonesia. Emerg Infect Dis 1999; 5: 585-8.

19.- Pape J, Verdier R, Boncy M, Boncy J, Johnson W. Cyclospora infection in adults infected with HIV. Clinical manifestations, treatment, and prophylaxis. Ann Intern Med 1994; 121: 654-7.

20.- Kumar S, Ananthan S, Lakshmi P. Intestinal parasitic infection in HIV infected patients with diarrhoea in Chennai. Indian J Med Microbiol 2002; 20: 88-91.

21.- Weitz JC, Botehlo R, Bryan R. Microsporidiosis en pacientes con diarrea crónica y SIDA, VIH asintomáticos y pacientes con diarrea aguda. Rev Méd Chile 1995; 123: 849-56.

22.- Connor B, Shlim D, Scholes J, Rayburn J, Reidy J, Rajah R. Pathologic changes in the small bowel in nine patients with diarrhea associated with a coccidia-like body. Ann Intern Med 1993; 119: 377-82.

23.- Behera B, Mirdha B, Makharia G, Bhatnagar S, Dattagupta S, Samantaray J. Parasites in patients with malabsorption syndrome: A clinical study in children and adults. Dig Dis Sci 2008; 53: 672-9.

24.- Yusuf T, Baron T. AIDS cholangiopathy. Curr Treat Options Gastroenterol 2004; 7: 111-7.

25.- Madrid V, Torrejón E, Rivera N, Madrid M. Ciclosporosis. Reporte de un caso clínico en Concepción, Chile. Rev Méd Chile 1998; 126 : 559-62.

26.- Jelinek T, Lotze M, Eichenlaub S, Löscher T, Nothdurft H. Prevalence of infection with Cryptosporidium parvum and Cyclospora cayetanensis among international travelers. Gut 1997; 41: 801-4.

27.- Verweij J, Laeijendecker D, Brienen E, van Lieshout L, Polderman A. Detection of Cyclospora cayetanensis in travellers returning from the tropics and subtropics using microscopy and real-time PCR. Int J Med Microbiol 2003; 293: 199-202.

28.- Long E, Ebrahimzadeh A, White E, Swisher B, Callaway C. Alga associated with diarrhea in patients with acquired immunodeficiency syndrome and in travelers. J Clin Microbiol 1990; 28: 1101-4.

29.- Bendall R, Lucas S, Moody A, Tovey G, Chiodini P. Diarrhoea associated with cyanobacterium-like bodies: a new coccidian enteritis of man. Lancet 1993; 341: 590-2.

30.- Junod C, Deluol A, Cosnes J, Bauer P. Cyclospora, nouvelle coccidie agent de diarrhées des voyageurs 11 observations. Presse Med 1994; 23: 1312 .

31.- Berlin O, Novak S, Porschen R, Long E, Stelma G, Schaeffer F. Recovery of Cyclospora organisms from patients with prolonged diarrhea. Clin Infect Dis 1994; 18: 606-9.

32.- Crowley B, Path C, Moloney C, Keane C. Cyclospora species-a cause of diarrhoea among Irish travelers to Asia. Ir Med J 1996; 89: 110-2.

33.- Gascón J, Corachan M, Bombi J, Valls M, Bordes J. Cyclospora in patients with traveler's diarrhea. Scan J Infect Dis 1995; 27: 511-4.

34.- Bernoux A, Libert J, Popoff D, Pays J. Cas groups d'infections gastro-intestinales a Cyclospora au retour d'un voyage en indonésie. BEH 1999; 42: 175-6.

35.- Gascón J, Álvarez M, Valls E, Bordas M, Jiménez de Anta T, Corachán M. Ciclosporiasis: estudio clinico-epidemiológico en viajeros con Cyclospora cayetanensis importada. Med Clin (Barc) 2001; 116: 461-4.

36.- Puente S, Morente A, García-Benayas T, Subirats M, Gascón J, González-Lahoz J. Cyclosporiasis: a point source outbreak acquired in Guatemala. J Travel Med 2006; 13: 334-7.

37.- Bourée P, Lancon A, Bisaro F, Bonnot G. Six human cyclosporiasis: with general review. J Egypt Soc Parasitol 2007; 37: 349-60.

38.- Ramírez-Olivencia G, Herrero MD, Subirats M, Rivas González P, Puente S. Brote de Cyclospora cayetanensis en viajeros a Cuba. Enferm Infecc Microbiol Clin 2008; 26: 558-60.

39.- Herwaldt B, Ackers M and the Cyclospora Working Group. An outbreak in 1996 of cyclosporiasis associated with imported raspberries. N Engl J Med 1997; 336: 1548-56.

40.- Döller P, Dietrich K, Filipp N, Brockmann S, Dreweck C, Vonthein R, et al. Cyclosporiasis outbreak in Germany associated with the consumption of salad. Emerg Infect Dis 2002; 8: 992-4.

41.- Rooney R, Cramer E, Mantha S, Nichols G, Bartram J, Farber J, Benembarek P. A review of outbreaks of waterborne disease associated with passenger ships: evidence for risk management. Public Health Rep 2004; 119: 427-34.

42.- Botero-Garcés J; Montoya-Palacio M, Barguil J, Castaño-González A. Brote epidémico por Cyclospora cayetanensis en Medellín. Rev Salud Pública 2006; 8 : 258-68.

43.- Eberhard M, Pieniazek N, Arrowood M. Laboratory diagnosis of Cyclospora infections. Arch Pathol Lab Med 1997; 121: 792-7. 
44.- Visvesvara G, Moura H, Kovacs-Nace E, Wallace S, Eberhard M. Uniform staining of Cyclospora oocysts in fecal smears by a modified safranin technique with microwave heating. J Clin Microbiol 1997; 35: 730-3.

45.- Galván-Díaz A, Herrera-Jaramillo V, SantosRodríguez Z, Delgado-Naranjo M. Coloraciones Ziehl-Neelsen y safranina modificadas para el diagnóstico de Cyclospora cayetanensis. Rev Salud Pública 2008; 10: 488-93.

46.- Abramowicz M. Drugs for parasitic diseases. Treat Guidel Med Letter 2007; 5 (Suppl): e1-15.

47.- Verdier R, Fitzgerald D, Johnson W, Pape J. Trimethoprim-sulfamethoxazole compared with ciprofloxacin for treatment and prophylaxis of
Isospora belli and Cyclospora cayetanensis infection in HIV-infected patients. A randomized, controlled trial. Ann Intern Med 2000; 132: 885-8

48.- Zimmer S, Schuetz A, Franco-Paredes C. Efficacy of nitazoxanide for cyclosporiasis in patient with sulfa allergy. Clin Infect Dis 2007; 44: 466-7. 